\title{
Adaptive partitioning approach to self-sustained smart grid
}

\author{
Youwei Jia ${ }^{1}$, Chun Sing Lai ${ }^{2}$, Zhao $X u^{1} \bowtie$, Songjian Chai ${ }^{1}$, Kit Po Wong ${ }^{3}$ \\ ${ }^{1}$ Department of Electrical Engineering, The Hong Kong Polytechnic University, Hong Kong SAR, People's Republic of China \\ ${ }^{2}$ Energy and Power Group, Department of Engineering Science, University of Oxford, Oxford, United Kingdom \\ ${ }^{3}$ School of Electrical, Electronics and Computer Engineering, The University of Western Australia, Perth, Australia \\ 凶-mail: eezhaoxu@polyu.edu.hk
}

\begin{abstract}
Effective network partitioning becomes an essential step to realise self-sustained smart grid, which serves as a prerequisite for 'self-healing' enabled decentralised control. Splitting the power network (PN) into areas is the last resort to avoid the spread of disruption and to maintain as many network survivals as possible. This study aims to resolve the issue of multi-objective PN partitioning by deploying a newly proposed hybrid approach concerning both real power balance and voltage profile. The proposed approach combines the Laplacian spectrum and self-organising map, which adaptively attains self-sustained network partitions on different operating conditions. The resultant partitions are characterised by the minimal intra-area real power imbalance with a healthy voltage profile. The authors experimentally evaluate the partitioning effectiveness and computational efficiency in several case studies including on the New England 39-bus, IEEE 118-bus, and Polish 2383-bus transmission systems.
\end{abstract}

\section{Introduction}

Power system security is a major concern at the present day due to the increasing complexities and uncertainties involved in system operation and control. The smart grid vision aims at developing the power system towards a 'self-healing' grid that is well integrated with advanced metering technologies, wide-area communication and automated controls. The benefits include fast decision-making, high controllability and system reliability. In general, a self-healing process comprises three phases [1]. First, system faults are timely and accurately monitored. Second, specific control actions are promptly taken to counteract system disturbance and isolate faulty parts of the network to avoid further spread of disruption. Finally, network reconfiguration and restoration will be in place and tune the entire system towards a stable and secure state. It is obvious that a suitable control scheme governing the whole system is of fundamental importance for achieving self-healing capability to cope with abnormal situations.

Admittedly, hierarchical centralised supervisory controls can be effective in a global perspective to achieve self-healing in small-scale power networks (PNs) but may be less efficient for large-scale systems [2], in which several profound complexities with respect to generation mix, load diversity and interconnected network topologies are involved. In some recent works, decentralised controls have been exploited and proven to be more tangible and manageable for practical applications in smart grids. In $[3,4]$, it is investigated that system data could be handled by the local agents without the need of centralised data acquisition and processing to achieve system monitoring and distributed control. However, the feasibility of controlling and recovering the network functionalities of large-scale PNs in a distributed manner is critically dependent on effective network partitioning, which still remains an open problem especially for large inter-connected PNs. In light of the above, it is mainly concerned in this paper to pursue self-sustained partitions with multiple optimisation objectives under different operating conditions. This work aims at laying a useful groundwork for smart grid planning and operation control with enabled self-healing capability. The detailed mechanisms of decentralised control and reconfigurations are beyond the scope of this paper.
As distinguished from existing partitioning works with sole objective, our study simultaneously takes into account of network connectivity, intra-area real power balance and voltage profile, which extensively addresses the prerequisites of self-healing capability. In particular, real and reactive power imbalances are jointly considered to formulate a multi-objective PN partitioning problem, of which the optimal partitions is the result of minimising real and reactive power exchange between adjacent areas. In a static sense of power system operation, minimising the intra-area real power imbalance (i.e. intra-area generation-load imbalance) can greatly assists in regulating frequency stability in each islands. In addition, sufficient reactive power supply within islands can avoid voltage instability and prevents the occurrence of undesirable electric components tripping events. Solely considering real or reactive power only may result in ineffective partitioning solutions for practical applications. For example, an optimal partitioning solution reflecting minimal generation-load imbalance do not necessarily guarantee healthy voltage profiles within each island. Nevertheless, solving multi-objective network partitioning problem is challenging and generally NP-hard. In view of the points discussed above, the major contributions of this paper can be summarised as follow:

- A hybrid approach combining specific PN Laplacians and self-organising map (SOM) algorithm is developed to capture eigenstructures directly with respect to multiple objectives.

- Pareto optimality can be achieved, in which multiple partitioning solutions are collectively provided. This can greatly facilitate decision-making of system operators.

- It is efficient to handle large-scale PNs by using the proposed partitioning approach, which is particularly tested on a Polish 2383-bus system.

- The partitions obtained in this paper are mainly based on steady state characteristics (i.e. real and reactive power flows). It is worth noting that the proposed method can be easily extended to fast-time-scale applications by incorporating specific weighted Laplacians with system dynamics.

The rest of this paper is organised as follows. In Section 2, we first review the existing methods of network partitioning for power 
system applications. In Sections 3 and 4, Laplacian spectrum with different attributes and detailed mechanism of SOM are introduced, respectively. Furthermore, the proposed heuristic algorithm to achieve Pareto optimality is presented in details. Experimental results are shown in Section 5. Section 6 concludes this paper.

\section{Network partitioning of power grids}

A power grid is generally composed of several synchronous interconnected sub-systems, which are referred as 'control areas'. Each of them is normally governed by the respective control entity (e.g. regional transmission organisation). These areas are traditionally formulated by only considering asset ownerships, local policies and regulations. Directly implementing such control areas is unable to fit with the time-varying operating conditions and diverse control requirements in modern power systems. In this regard, smart grid operation and control necessitate adaptive and efficient PN partitions considering specific engineering constraints.

Network partitioning deployed in power engineering is not novel and many research efforts have been made to handle different applications associated with big data and complex topological structures, such as resource adequacy assessments [5], area-based voltage stability assessment and control [6], zonal pricing in electricity market [7], etc. Partitioning methods are also employed in system planning and operational security analysis. For example, Donde et al. [8] address generation-load imbalance problems based on steady or quasi-steady state analysis; several studies investigate the synchronisation of complex PNs (e.g. [9]) or slow coherency (e.g. [10]) to identify the dynamic coherent sub-networks based on dynamic analysis. Moreover, Xie et al. [11] proposed a useful graph theory based approach to find out the optimal network partitions for the PMU placement. From the perspective of the network topology, this approach is effective to provide the structural information to the system operators. In general, two broad categories of partitioning methods have been reported in power system literature - mathematical modelling based optimisation methods and network Laplacians based spectral methods. The first class of methods typically formulate the network partitioning as a mix-integer linear/non-linear optimisation problem and have it solved through well-established commercial software packages (e.g. AMPL, CPLEX, etc.) or intelligent optimisation algorithms. With advanced global optimisation algorithms involved, this type of methods can normally provide guarantees regarding the optimality. However, the convergence speed is essentially dependent on the modelling complexity. In the existing literature, most of such works were proposed with different levels of modelling simplification to make the problem be tractable. For example, Sun et al. [12] proposed a two-step partitioning method for system restoration, in which the network topology is simplified to reduce the computational burden. Ding et al. [13] proposed an efficient mix-integer linear programming based partitioning approach for controlled islanding operation, whereas only DC power flow is formulated and voltage constraints are neglected. In considering multiple attributes/partitioning objectives, Cotilla-Sanchez et al. [14] proposed an efficient method by combining all attributes into one objective. Partitioning solutions can be easily obtained by employing evolutionary algorithms, but the performance of this kind of methods can be sensitive to single objective formulation and the compatibilities of all attributes remain questionable. Instead of complex modelling of network topologies and engineering constraints, weighted Laplacians based spectral methods (e.g. [15]) can directly yield partitions from eigenstructures of Laplacian matrices. Even though multi-objectives can be effortlessly incorporated into this type of methods by taking into account, e.g. combined weights on each branch [16], partitioning solutions obtained by normal spectral clustering might be semi-optimal and no Pareto sets can be provided. Therefore, it is noted that not every partitioning method is equally good or suitable for a specific engineering application. Moreover, to the authors' best knowledge, most of the existing methods are less effective to target multi-objective partitioning.
In our proposed approach, weighted Laplacians represented by real and reactive power distribution are collectively considered to measure self-sustainability of each partitioning area. SOM with an advanced visualisation technique is used as a tool to produce partitions from different eigenstructures. In order to achieve Pareto optimality, a heurist algorithm to manage and tune the combined eigenspace of weighted Laplacians is proposed in this paper. Detailed mechanisms of the proposed partitioning approach will be illustrated in the forthcoming sections.

\section{Laplacian spectrum of PNs}

\subsection{Preliminaries of spectral representation}

The topological properties of a PN can be extracted from the network Laplacian spectrum. Unnormalised Laplacian matrix is expressed as

$$
\boldsymbol{L}=D-W
$$

where $\boldsymbol{W}=\left(\omega_{i j}\right)_{i, j=1,2, \ldots, N}$ is the weighted power flow matrix, which can be formulated in terms of the real and reactive power $[17,18]$. According to the well-established power flow equations, the weights of the transmission lines are assigned as the real and reactive power flows through them.

$$
\begin{gathered}
\omega_{i j}^{\mathrm{MW}}=P_{i j}=U_{i} U_{j}\left(G_{i j} \cos \theta_{i j}+B_{i j} \sin \theta_{i j}\right) \\
\omega_{i j}^{\mathrm{MVar}}=Q_{i j}=U_{i} U_{j}\left(G_{i j} \sin \theta_{i j}-B_{i j} \cos \theta_{i j}\right)
\end{gathered}
$$

where $P_{i j}$ and $Q_{i j}$ represent the real and reactive power flow on line $(i$, $j) . U_{i}$ is the bus voltage of bus $i . \theta_{i j}$ is the phase-angle difference between bus $i$ and $j . Y_{i j}=G_{i j}+j B_{i j}, Y_{i j}$ is the admittance of line $(i, j) . \boldsymbol{D}$ is a diagonal matrix and each of the diagonal element indicates the contribution of the corresponding bus in handling power traffic in the PN [17], which is formulated as

$$
\boldsymbol{D}(\mathcal{G})=\operatorname{diag}\left\{d_{1}, d_{2}, \ldots, d_{|\mathrm{N}|}\right\}, \quad d_{i}=\sum_{j=1}^{N} \omega_{i j}
$$

There are two forms of normalised Laplacian matrix. Both are closely related to each other.

$$
\begin{gathered}
\boldsymbol{L}_{\mathrm{sym}}=D^{-1 / 2} L D^{-1 / 2}=I-D^{-1 / 2} W D^{-1 / 2} \\
\boldsymbol{L}_{\mathrm{rw}}=D^{-1} L=I-D^{-1} W
\end{gathered}
$$

where $\boldsymbol{L}_{\text {sym }}$ is a symmetric matrix, and $\boldsymbol{L}_{\mathrm{rw}}$ is closely related to a random walk. $\boldsymbol{L}_{\mathrm{sym}}$ and $\boldsymbol{L}_{\mathrm{rw}}$ are positive semi-definite and have $N$ non-negative real-valued eigenvalues. The multiplicity of zero eigenvalue is equal to the number of connected sub-graphs [19].

Mathematically speaking, the clustering information on the loosely coupled connected components in a graph is spanned over the eigenvectors of Laplacian matrix. Davis-Kahan theorem [20] introduced below reveals the relationship between clustering performance and the eigengap, which is defined as the largest gap between two neighbouring eigenvalues.

Theorem (Davis-Kahan): Given that symmetrical matrices $\boldsymbol{A}, \boldsymbol{H} \in R^{N \times N}$. Let $S \subset \boldsymbol{R}$ be an interval. The set of eigenvalues of $\boldsymbol{A}$ which fall into the interval $\boldsymbol{S}$ is denoted by $\Lambda_{S}(A) . \boldsymbol{V}$ is the corresponding eigenspace of $\Lambda_{S}(A)$. Consider perturbed $\tilde{A}=A+H$, and $\tilde{V}$ is the analogous quantity for $\tilde{A}$. The distance between $\boldsymbol{S}$ and the spectrum of $\boldsymbol{A}$ outside of $\boldsymbol{S}$ is

$$
\delta=\min \{|\lambda-s| ; \lambda \notin S, s \in S\}
$$

Thus the distance $d(V, \tilde{V})=\|\sin \Theta(V, \tilde{V})\|$ is bounded by

$$
d(V, \tilde{V}) \leq\|H\| / \delta
$$


where $\sin \Theta$ denotes a diagonal matrix with the canonical angles on the diagonal.

Given that $\tilde{\boldsymbol{L}}=\boldsymbol{L}+H$, where $\boldsymbol{L}$ is the Laplacian matrix of a disconnected graph, if both $\Lambda_{S}(\boldsymbol{L})$ and $\Lambda_{S}(\tilde{\boldsymbol{L}})$ are within $\boldsymbol{S}$, the eigenspace of $\boldsymbol{L}$ and $\tilde{\boldsymbol{L}}$ are approximately close to each other. Since their distance is bounded by $\|H\| / \delta$ according to the DavisKahan theory, hence the degree of such 'approximation' is strongly related to the perturbation $\|H\|$. Given that $\boldsymbol{S}$ is set by $\left[0, \lambda_{k}\right]$, then $\delta$ is consistent with the eigengap $\left|\lambda_{k+1}-\lambda_{k}\right|$.

Based on the Davis-Kahan theorem, one can safely draw the conclusion that the larger the eigengap $\left|\lambda_{k+1}-\lambda_{k}\right|$ is, the more clustering information can be extracted from the first $k$ eigenvectors. This conclusion is widely used in classical spectral clustering and forms the theoretical basis in our study for the construction of combined eigenspace, which will be introduced in the forthcoming subsection.

\subsection{Spectral properties of a PN}

By recalling the partitioning objective as mentioned in Section 2, we aim at approaching the optimal cut which minimises both real and reactive power exchanges among all clusters. It is obvious that less power exchange among different clusters reflects a better 'self-sufficient' condition. However, it should be noted that an optimal partition considering only real power flows may lead to an unsatisfactory voltage profile within sufficient reactive power support. Likewise, network partition with respect to reactive power is unable to guarantee a small real power generation-load imbalance.

In this paper, we mathematically formulate the concerning PN partitioning problem as a multi-objective minimal Ncut to minimise the inter-cluster similarities and maximise the intra-cluster affinities. Ncut (first introduced in [21]) is defined as

$$
N \operatorname{cut}\left(C_{1}, \ldots, C_{k}\right)=\sum_{i=1}^{k} \frac{\operatorname{cut}\left(C_{i}, \bar{C}_{i}\right)}{\operatorname{vol}\left(C_{i}\right)}
$$

where $\bar{C}_{i}$ is the complement of $C_{i}$

$$
\operatorname{cut}\left(C_{i}, \bar{C}_{i}\right)=\sum_{i \in C_{i}, j \in \bar{C}_{i}} \omega_{i j}, \quad \operatorname{vol}\left(C_{i}\right)=\sum_{i \in C_{i}} \sum_{j=1}^{|N|} \omega_{i j}
$$

In particular, the formulated Min- $N$ cut minimises the inter-cluster real and reactive power flow exchanges and maximises the intra-cluster self-contained capacities. Such formulation effectively avoids 'one-single-node cluster' phenomena, in which normalised Laplacian matrix is selected as the topological representation. Between $\boldsymbol{L}_{\mathrm{sym}}$ and $\boldsymbol{L}_{\mathrm{rw}}$, the proposed approach is in favour of $\boldsymbol{L}_{\mathrm{rw}}$ due to its better experimental performance than that of $\boldsymbol{L}_{\mathrm{sym}}$. This may be because eigenvectors of $\boldsymbol{L}_{\mathrm{sym}}$ are additionally multiplied by $D^{1 / 2}$ and this undesirably influences its eigenstructure. In addition, there is no computational advantage when using $\boldsymbol{L}_{\text {sym }}$. Hence $\boldsymbol{L}_{\mathrm{rw}}$ is selected in our approach.

Normalised Laplacian matrices $\boldsymbol{L}_{\mathrm{MW}}$ and $\boldsymbol{L}_{\mathrm{MVar}}$ are constructed, in which the weights of branches are assigned by the absolute value of real and reactive power flows, respectively. According to the discussion in Section 3.1, eigengaps exist in both $\boldsymbol{L}_{\mathrm{MW}}$ and $\boldsymbol{L}_{\mathrm{MVar}}$ expose the clustering information contained in the first few eigenvectors. The clustering boundaries could be easily identified with a large eigengap. On the contrary, the existing clusters are overlapping and boundaries are ambiguous.

In considering both real and reactive power flows, a combined eigenspace comprising eigenvectors of $\boldsymbol{L}_{\mathrm{MW}}$ and $\boldsymbol{L}_{\mathrm{MVar}}$ is constructed

$$
V_{\text {comb }}:=\left[V_{\mathrm{MW}} \mid V_{\mathrm{MVar}}\right]
$$

where $V_{\mathrm{MW}}$ and $V_{\mathrm{MVAr}}$ consist of selective number of eigenvectors of $L_{\mathrm{MW}}$ and $L_{\mathrm{MVAr}}$, respectively. The combined eigenspace can be adjusted in a heuristic way by containing different clustering information of real and reactive power. $k$ is termed as a heuristic indicator in this paper and formulated as

$$
\begin{gathered}
k_{\mathrm{MW}}=\arg \max \left\{\left|\lambda_{k+1}-\lambda_{k}\right| ; \lambda \in \Lambda_{S}\left(\boldsymbol{L}_{\mathrm{MW}}\right)\right\} \\
k_{\mathrm{MVar}}=\arg \max \left\{\left|\lambda_{k+1}-\lambda_{k}\right| ; \lambda \in \Lambda_{S}\left(\boldsymbol{L}_{\mathrm{MVar}}\right)\right\}
\end{gathered}
$$

where $\boldsymbol{S}$ is a predefined interval. Algorithm 1 illustrates (see Fig. 1) the procedure of constructing $\boldsymbol{V}_{\mathbf{c o m b}}$

Clustering the points $\left(y_{i}\right)_{i=1, \ldots|N|}:=\left[x_{1}^{i}, \ldots, x_{k_{\mathrm{MW}}+k_{\mathrm{MVar}}}^{i}\right]$ obtained from the eigenspace is an essential step to identify the partitioning boundaries. Instead of using $k$-means algorithm directly, which has been found to be inaccurate and suffer from local minima for large networks, SOM algorithm with advanced visualisation technique is used in our approach to adaptively handle massive network data. Full details of SOM based partitioning algorithm are discussed in Section 4.

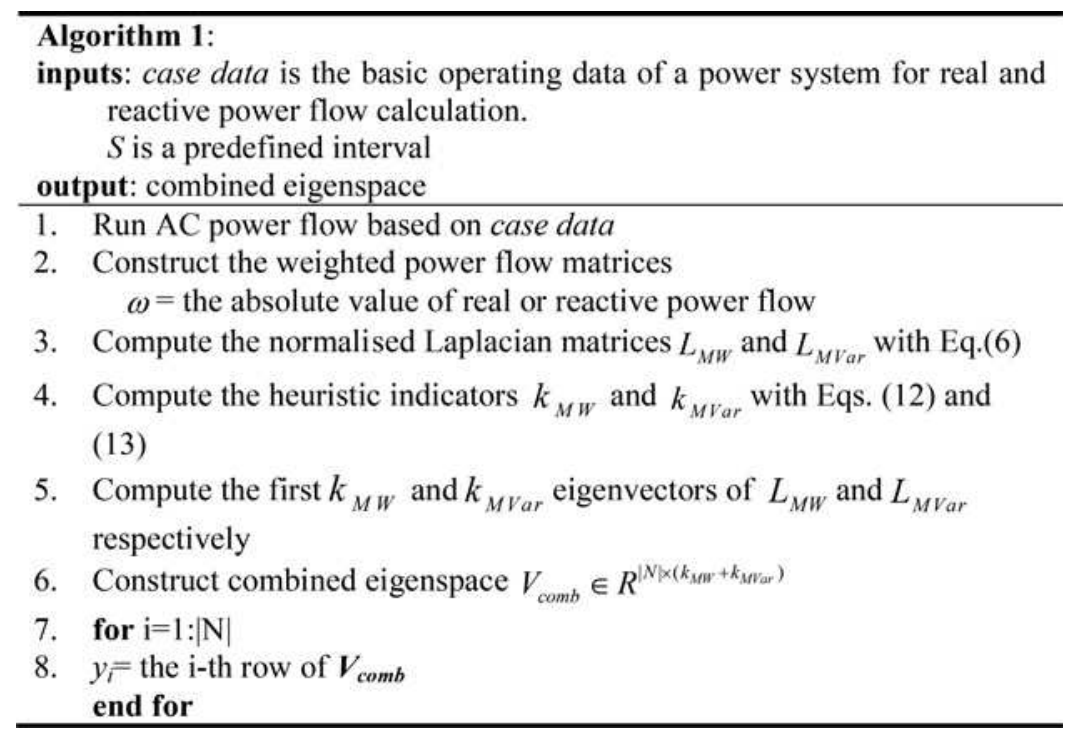

Fig. 1 Construction of combined eigenspace 


\section{Proposed methodology of adaptive $K$-way network partitioning}

Multi-objective partitioning problem is known to be NP-complete. Traditional spectral clustering fails to tackle such problems while some of its variations have been proposed to handle multi-objectives, e.g. [22] by using collective weights integrating all concerned aspects. However, this kind of approaches is highly sensitive to predetermined preference factors when forming integrated weights.

The proposed method consists of two parts including automated clustering based on SOM and multi-objective optimisation. The flowchart shown in Fig. 2 illustrates the basic procedures of the proposed approach.

\subsection{Automated clustering based on SOM}

SOM (also called as Kohonen Map) [23] is a widely used tool for cluster extraction, visualisation and data mining. SOM is an unsupervised, competitive artificial neural network that produces topology-preserving mappings of data spaces utilising a self-organising learning algorithm. This algorithm can project high-dimensional data $\mathcal{Y} \subset \boldsymbol{R}^{\text {d }}$ onto a low-dimensional (typically one or two-dimensional) fixed lattice of weighted neurons denoted as $\mathcal{K}$. The classical SOM algorithm is briefly explained as follows.

The weights (denoted as $W_{i}^{\text {neu }}:=\left[w_{1}, \ldots, w_{\left.\mid W_{\text {neu }}\right]}\right]$ ) of each neural unit in the Kohonen Map $\mathcal{K}$ are trained through a competitive learning process. The best matching unit is determined by the minimal Euclidean norms for a given data sample $y \in \mathcal{Y}$,

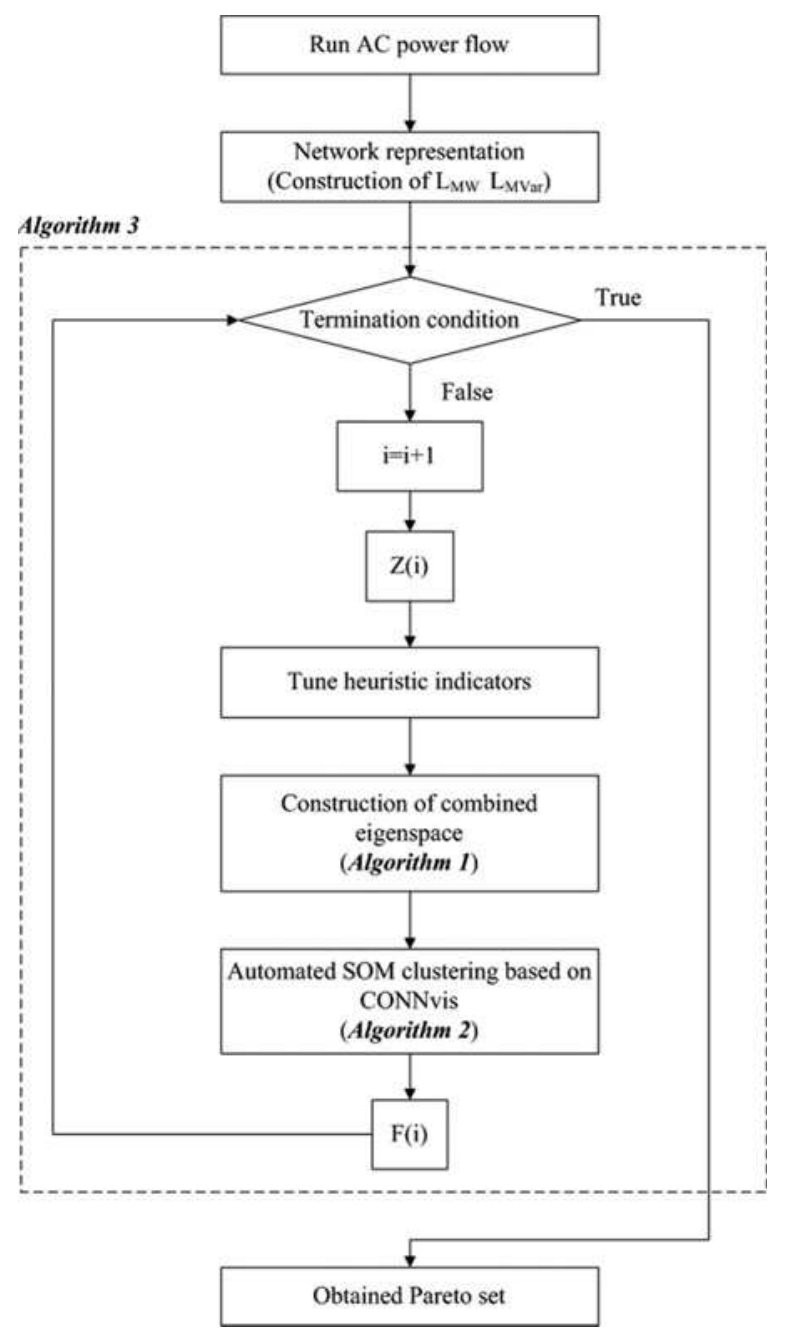

Fig. 2 Flowchart of the proposed partitioning approach such that

$$
\left\|y-W_{\text {best }}^{\mathrm{neu}}\right\| \leq\left\|y-W_{i}^{\mathrm{neu}}\right\| \quad \forall i \in \mathcal{K}
$$

The weights of each neuron are updated based on the following rule

$$
W^{\text {neu }}(t+1)=W^{\text {neu }}(t)+\eta(t) \Theta[\sigma(t)]\left(y-W^{\text {neu }}\right)
$$

where $\eta(t)$ is learning rate which exponentially decreases with $t . \Theta(\cdot)$ is the neighbourhood function and $\sigma(t)$ represents the neighbourhood size. The weight vector of each neuron is the centroid of its receptive field (RF).

SOM has high capability of extracting data structures on the condition of cluster-overlaps. In our work, a hierarchical agglomerative clustering algorithm based on SOM is applied to the multi-objective partitioning problem. Geometric representations (provided by Laplacian spectrum) of PNs serve as the inputs for SOM training. An effective visualisation technique (i.e. CONNvis [24]) is utilised to achieve automated clustering for large dataset and diverse cluster sizes. CONNvis is advantageous over other visualisation methods (e.g. Euclidean distance between quantisation neurons [25], neighbourhood of SOM [26], etc.) to handle overlapping clusters. This automated clustering algorithm is summarised in (see Fig. 3).

(i) Construct combined eigenspace $\boldsymbol{V}_{\text {comb }}$. Each row of $\boldsymbol{V}_{\text {comb }}$ serves as an input for SOM training.

(ii) A $t \times t$ Kohonen map is constructed and trained through a competitive training process. Selecting the number of neurons in Kohonen map follows the rule that $t^{2}$ should be significantly less than the number of data samples whilst greater than the expected number of clusters [24].

(iii) Connectivity matrix contains two important clustering information, namely neighbourhood relations in datasets and detailed data distribution [27]. It can be expressed as

$$
\operatorname{CONN}(i, j)=\left|R F_{i j}\right|+\left|R F_{j i}\right|
$$

where $R F_{i j}=\left\{y \in R F_{i}:\left\|y-W_{j}\right\| \leq|| y-W_{l} \| \quad \forall l \neq i\right\}$, and $\left|R F_{i j}\right|$ indicates the number of data vectors in $R F_{i j}$.

(iv) Similarities between neurons are determined by the local data distribution. Mathematically speaking, similarity matrix is initialised as connectivity matrix. $\boldsymbol{S}=\mathbf{C O N N}$.

(v) In each iterative step, the most similar pair of clusters $\boldsymbol{C}_{\boldsymbol{i}}$ and $\boldsymbol{C}_{\boldsymbol{j}}$ (which may include one or more neurons) is merged into a new one which is referred as cluster $\boldsymbol{p} . \boldsymbol{S}(\boldsymbol{p}, \boldsymbol{q})$ is formulated as

$$
\boldsymbol{S}(\boldsymbol{p}, \boldsymbol{q})=\left[\left|C_{i}\right| /\left(\left|C_{i}\right|+\left|C_{j}\right|\right)\right] S(i, q)+\left[\left|C_{j}\right| /\left(\left|C_{i}\right|+\left|C_{j}\right|\right)\right] S(j, q)
$$

The corresponding rows and columns of the most similar pair in $S$ are omitted accordingly. After $t^{2}-1$ iterations, all neurons are merged into one cluster. CONN linkage is calculated in each step, which is formulated as

$$
\text { conn_index }=\text { intra_conn } \times(1-\text { inter_conn })
$$

where

$$
\begin{aligned}
& \text { intra_conn }=\frac{1}{R} \sum_{r=1}^{R} \frac{\sum_{i, j \in C_{r}}\left|R F_{i j}\right|}{\sum_{i \in C_{r}}\left|R F_{i}\right|} \\
& \text { inter_conn }=\frac{1}{R} \sum_{r=1}^{R} \max \frac{\sum_{i \in C_{r}, j \in C_{l}}\left|R F_{i j}\right|}{\sum_{i \in B_{r l}} \operatorname{CONN}(i, j)}
\end{aligned}
$$

$R$ is the number of clusters. $B_{r l}$ is the set of neurons in cluster $r$ that are directly connecting to the neurons in cluster $l$. 


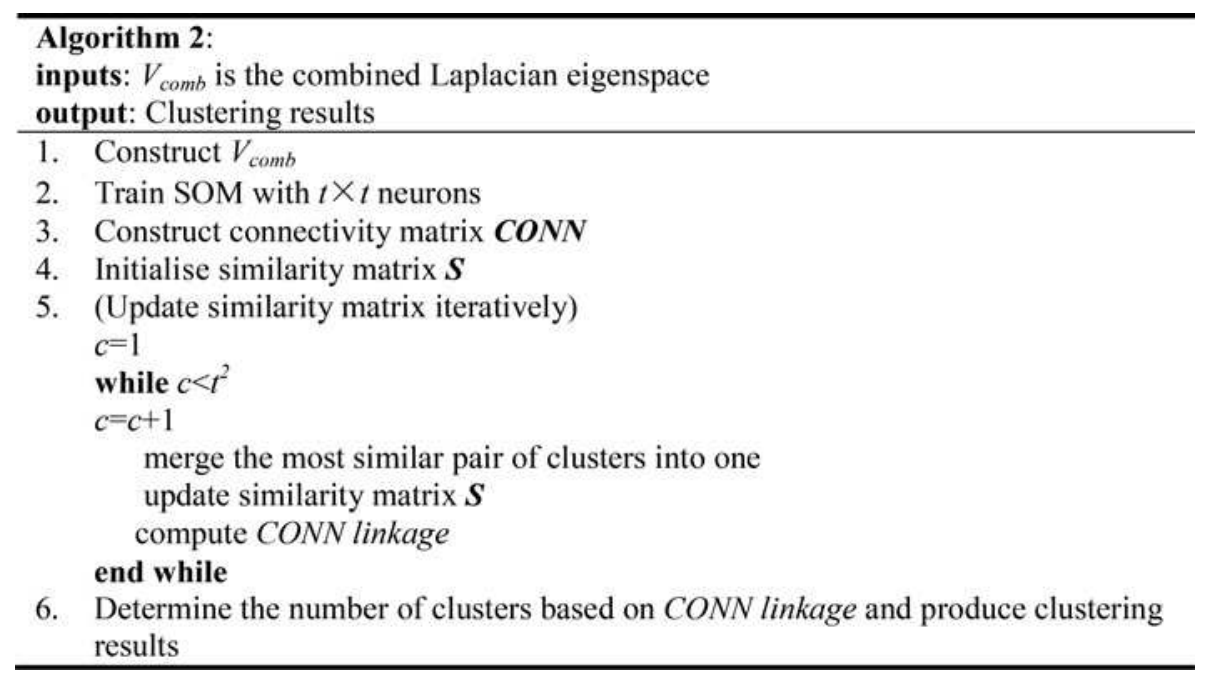

Fig. 3 Automated clustering based on SOM

(vi) The maximum value of conn_index is 1 , which indicates that all clusters are clearly separated. Based on the analysis in [24], higher conn_index value reflects better clustering results. In the proposed study, the number of clusters is determined according to the maximum conn_index value. Since $\boldsymbol{R}=1$ is meaningless for the proposed partitioning problem, $\boldsymbol{R}$ is bounded within $\left[2, \boldsymbol{R}_{\mathbf{m a x}}\right]$, where $\boldsymbol{R}_{\mathbf{m a x}}$ is the maximum number of acceptable clusters in a specific PN.

This algorithm has merits that no prior knowledge of cardinality of clusters is needed and obtained clusters can be of unequal sizes [28]. Since training set of SOM can be adjusted by $k_{\mathrm{MW}}$ and $k_{\mathrm{MVar}}$, this algorithm is capable of approaching a trade-off partitioning by considering both real and reactive power flows.

\subsection{Pareto optimality}

As discussed in previous sections, combined eigenspace constructed by different pairs of $k_{\mathrm{MW}}$ and $k_{\mathrm{MVAr}}$ can significantly influence the clustering results. Therefore, $k_{\mathrm{MW}}$ and $k_{\mathrm{MVAr}}$ can be used to adjust the clustering results regarding real and reactive power. For example, if more effective clustering information based on reactive power flow is contained in $V_{\text {comb }}$, clustering boundaries would be 'dragged' towards optimal reactive power partition. As a result of continuous adjustments of $k_{\mathrm{MW}}$ and $k_{\mathrm{MVAr}}$, one enclosing with more clustering information would 'prevail' over another.
Based on the analysis above, a heuristic method is proposed to solve the following bi-objective optimisation problem

$$
\min \left\{f_{1}: \sum_{r=1}^{R}\left|g_{r}-l_{r}\right|, f_{2}: \sum_{r=1}^{R}\left|\Delta v_{r}\right|\right\}
$$

where $g_{r}$ and $l_{r}$ are the total real power generation and load in cluster $r$, respectively. $\triangle v_{r}$ denotes the voltage violation in cluster $r$. In practice, voltage of each bus should be maintained within tolerance (i.e. $\pm 0.05 \mathrm{pu}$ ). In this paper, the voltage issue is simplified based on the assumption that only limited reactive power compensation is locally available on each bus.

The proposed approach aims to find out solutions of Pareto-optimal front and maintain a good spread of solutions on this front. Inspired by NSGA-II framework [29], a tailor-made algorithm for network partitioning is developed based on fast non-dominated sorting and crowding distance [29], which is illustrated in Fig. 4.

(i) $U(\bullet)$ represents a string of $m$ individuals. Each individual is a pair of heuristic indicators.

$$
U(\cdot)=\left[u_{1}, u_{2}, \ldots, u_{m}\right], \quad \text { where } u=\left[\begin{array}{c}
k_{\mathrm{MW}} \\
k_{\mathrm{MVar}}
\end{array}\right]
$$

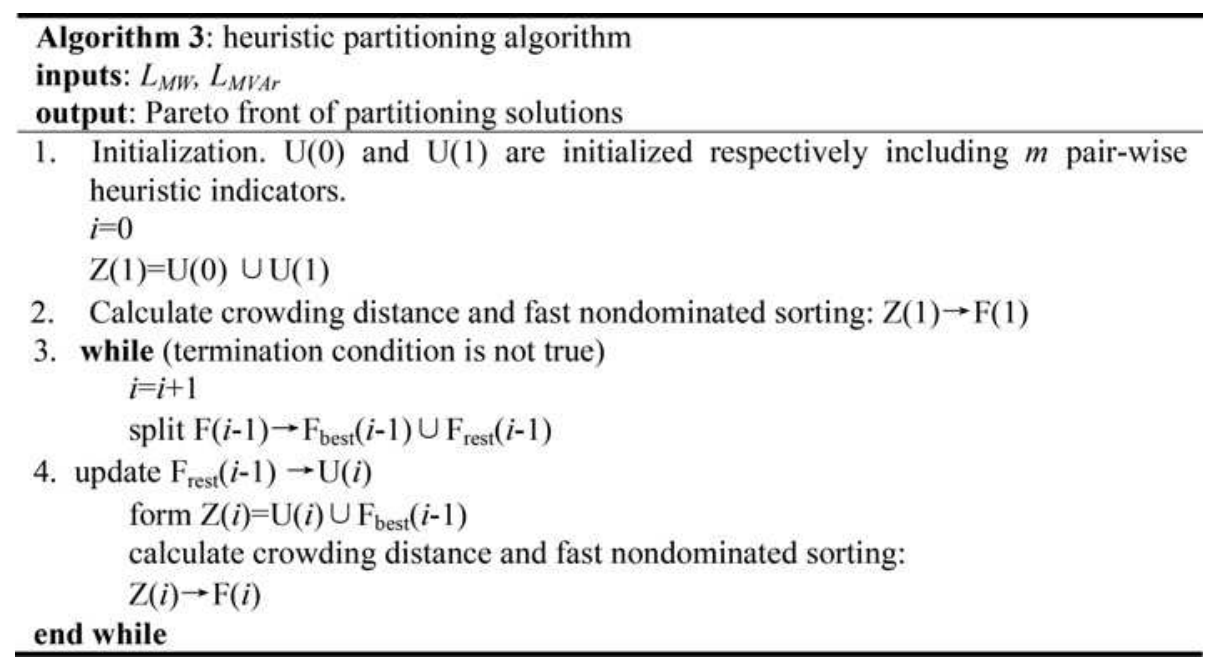

Fig. 4 Heuristic partitioning algorithm 


\begin{tabular}{|c|c|c|}
\hline 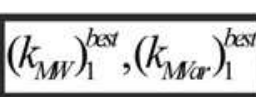 & $\left(k_{M W}\right)_{m+1}^{r e s t},\left(k_{M V a r}\right)_{m+1}^{r e t}$ & $\left(k_{M V}\right)_{1},\left(k_{M V a r}\right)_{1}$ \\
\hline 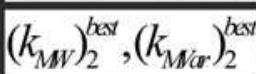 & $\left(k_{M W}\right)_{m+2}^{r e s t},\left(k_{M V a r}\right)_{m+2}^{r \text { rest }}$ & $\sqrt{\left(k_{M V}\right)_{2},\left(k_{M V r}\right)_{2}}$ \\
\hline$\cdots \cdots$ & $\cdots \cdots$ & $\ldots \ldots$ \\
\hline 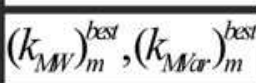 & $\left(k_{M W}\right)_{2 m}^{r s s t},\left(k_{M V a r}\right)_{2 m}^{r e s t}$ & $\overline{\left(k_{M W}\right)_{m},\left(k_{\text {MVar }}\right)_{m}}$ \\
\hline
\end{tabular}

Fig. 5 Schematic diagram of updating process

The heuristic indicator is randomly initialised in a given acceptable range $\left[1, \boldsymbol{k}_{\mathbf{m a x}}\right]$, which can be formulated as

$$
k=\left\lfloor 1+k_{\max } \cdot \gamma\right\rfloor
$$

where $\gamma$ is a random number within [0,1]. $U(0)$ and $U(1)$ are randomly initialised without duplicate individuals. $Z(1)$ is a combination of $U(0)$ and $U(1)$.

(ii) In this step, all individuals in $Z(1)$ are evaluated through the partitioning algorithm proposed in Section 4.1. Each partitioning solution is associated with a pair of heuristic indicators. Z(1) is first sorted by fast non-dominated sorting algorithm with total complexity of $O\left(2 \mathrm{~m}^{2}\right)$. Crowding distance is calculated between neighbouring non-dominated solutions. All of such solutions are subsequently re-sorted and re-arranged according to crowding distance ranking. High-ranking ones will lead to update the offspring populations.

(iii) In our approach, the termination criterion is $i \leq\left(k_{\mathrm{MW}} k_{\mathrm{MVar}}\right)_{\max } / 2 m . F(i)$ is obtained by sorting $Z(i) . F(i)$ is truncated into two parts with equal size. The first part consists of best individuals, which is denoted as $F_{\text {best }}(i)$. The rest is denoted as $F_{\text {rest }}(i)$. In the present application, it is defined that the heuristic algorithm converges as long as the rate of change of fitness values reaches a predefined tolerance (e.g. $2 \%$ in case studies in Section 5). iv. The schematic diagram of updating process is shown in Fig. 5.

The updating operator is formulated as

$$
\begin{array}{ll}
k_{j}=\left\lfloor k_{m+j}^{\text {rest }}+\left(k_{j}^{\text {best }}-k_{m+j}^{\text {rest }}+2\right) \cdot \gamma\right\rfloor, & k_{j}^{\text {best }} \geq k_{m+j}^{\text {rest }} \\
k_{j}=\left\lfloor k_{m+j}^{\text {rest }}+\left(k_{j}^{\text {best }}-k_{m+j}^{\text {rest }}-2\right) \cdot \gamma\right\rfloor, & k_{j}^{\text {best }}<k_{m+j}^{\text {rest }}
\end{array}
$$

where $\gamma$ is a random number ranged within $[0,1)$. $k_{j}$ is allowed to be assigned with a random value ranged within $\left[k_{m+j}^{\text {rest }}, k_{j}^{\text {best }}+1\right]$ or $\left[k_{m+j}^{\text {rest }}-1, k_{j}^{\text {best }}\right]$. This enables the proposed algorithm to explore and exploit the search space in an efficient way. $Z(i)$ incorporates $U(i)$ and $F_{\text {best }}(i-1)$ to strengthen the elitism throughout the whole process. $F(i)$ is obtained by rearranging $Z(i)$ according to crowding distance and fast non-dominated sorting.

\subsection{Fuzzy decision-making}

Given no prior knowledge for the selection of candidate partitions in Pareto set, un-weighted fuzzy logic decision-making strategy [30] is employed to yield the best trade-off solution. It is worth noting that other decision-making criterions can also be considered with particular preferences.

In this paper, we assume that the preferences of minimising voltage violation and generation-load imbalance are unbiased. Fuzzy logic decision-making is formulated as follows.

Given a Pareto set $S_{\text {pareto }}$ for pair-wise objectives, the fuzzy membership is defined as

$$
\mu_{i}=\frac{f_{i}^{\max }-f_{i}}{f_{i}^{\max }-f_{i}^{\min }}, \quad i=1,2
$$

The normalised membership for each solution is expressed as

$$
\mu(j)=\frac{\sum_{i=1}^{2} \mu_{i}(j)}{\sum_{j=1}^{\left|S_{\text {pareto }}\right|} \sum_{i=1}^{2} \mu_{i}(j)}
$$

According to the fuzzy logic principle [30], the trade-off (most satisfactory) solution is selected with the maximum fuzzy membership value.
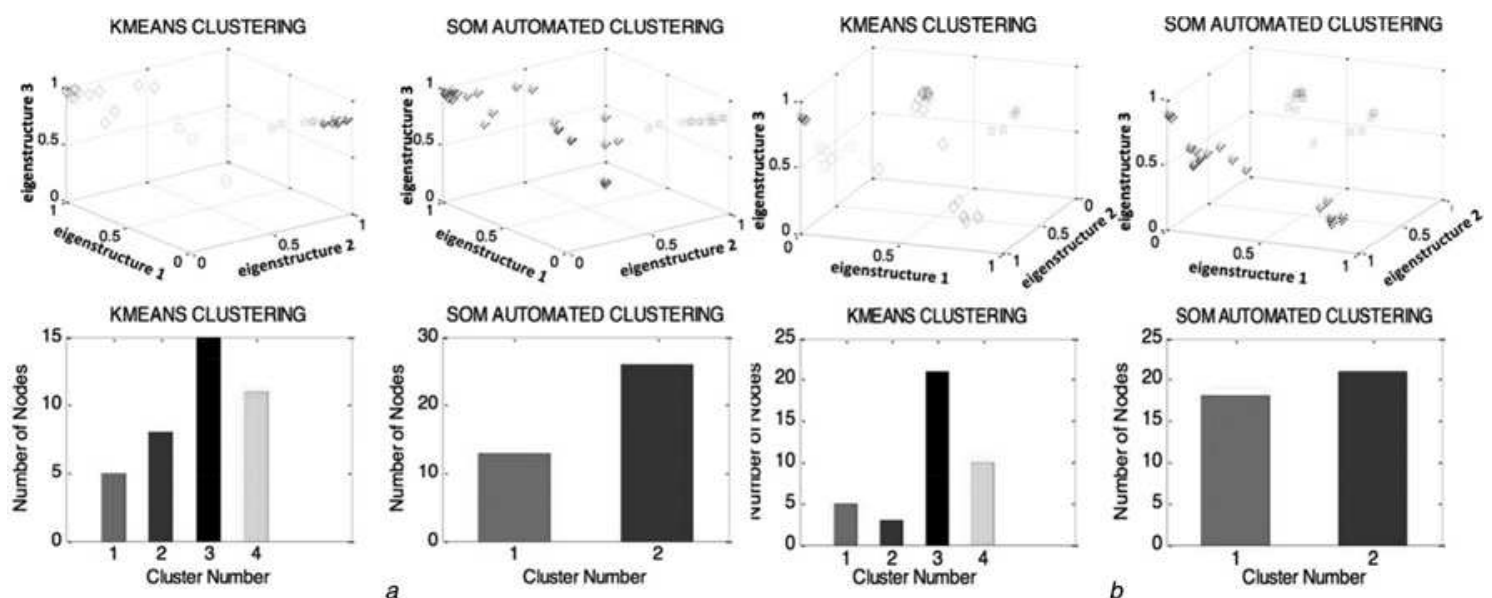

Fig. 6 Comparative experiment results

$a$ Clustering results based on SOM $\left(k_{\mathrm{MW}}=1, k_{\mathrm{MVAr}}=2\right)$ and $k$-means in normal operating condition $b$ Clustering results based on SOM $\left(k_{\mathrm{MW}}=3, k_{\mathrm{MVAr}}=2\right)$ and $k$-means in normal operating condition 
Table 1 Clustering performance based on SOM and $K$-means (New England 39-bus System)

\begin{tabular}{lcccc}
\hline SOM & $\begin{array}{c}\text { Real power } \\
\text { imbalance, MW }\end{array}$ & $\begin{array}{c}\text { Voltage } \\
\text { violation, p.u }\end{array}$ & $\begin{array}{c}\text { Buses } \\
\text { (abnormal } \\
\text { voltage) }\end{array}$ \\
\hline k-means & $\begin{array}{c}k_{\mathrm{MW}}=1 \\
k_{\mathrm{MVAr}}=2 \\
k_{\mathrm{MW}}=3\end{array}$ & 147.259 & 0.0036 & 36 \\
$k_{\mathrm{MVAr}}=2$ & 4.2 & 0.0212 & $4,7,8,36$ \\
$k_{\mathrm{MW}}=1$ & 170.759 & 0.0288 & $4,15,24,36$ \\
$k_{\mathrm{MVAr}}=2$ & & 195.1 & 0.0246 & $4,7,8,24,36$ \\
& $k_{\mathrm{MW}}=3$ & & & \\
\hline$k_{\mathrm{MVAr}}=2$ & & &
\end{tabular}

Table 2 Partitioning results (New England 39-bus system)

\begin{tabular}{|c|c|c|c|c|}
\hline $\begin{array}{l}\text { Heuristic } \\
\text { partitioning }\end{array}$ & $\begin{array}{l}\text { Partitioning } \\
\text { alternatives (with } \\
\text { cluster sizes) }\end{array}$ & $\begin{array}{c}\text { MW } \\
\text { imbalance, } \\
\text { MW }\end{array}$ & $\begin{array}{l}\text { Voltage } \\
\text { violation, } \\
\text { p.u }\end{array}$ & $\mu$ \\
\hline \multicolumn{5}{|c|}{ Normal operating condition } \\
\hline \multirow[t]{2}{*}{ SOM } & $\begin{array}{l}\text { Cluster 1:14 } \\
\text { Cluster 2:25 }\end{array}$ & 0 & 0.0036 & $0.9241 *$ \\
\hline & $\begin{array}{l}\text { Cluster 1:7 } \\
\text { Cluster 2:32 }\end{array}$ & 46.6 & 0.003 & 0.0759 \\
\hline \multirow[t]{3}{*}{$k$-means } & $\begin{array}{c}\text { Cluster 1:15 } \\
\text { Cluser2:24 }\end{array}$ & 4.2 & 0.02117 & 0.5044 \\
\hline & $\begin{array}{c}\text { Cluster 1:6 } \\
\text { Cluster 2:13 } \\
\text { Cluster 3:14 } \\
\text { Cluster } 4: 6\end{array}$ & 420 & 0.007012 & 0.4063 \\
\hline & $\begin{array}{c}\text { Cluster 1:7 } \\
\text { Cluster 2:13 } \\
\text { Cluster 3:14 } \\
\text { Cluster } 4: 5\end{array}$ & 543.6 & 0.0036 & 0.0894 \\
\hline \multicolumn{5}{|c|}{ Heavy load operating condition } \\
\hline \multirow[t]{2}{*}{ SOM } & $\begin{array}{l}\text { Cluster 1:13 } \\
\text { Cluster } 2: 26\end{array}$ & 309.5 & 0.0036 & $0.8926^{*}$ \\
\hline & $\begin{array}{l}\text { Cluster 1:12 } \\
\text { Cluster 2:27 }\end{array}$ & 353.5 & 0.0027 & 0.1074 \\
\hline \multirow[t]{3}{*}{$k$-means } & $\begin{array}{c}\text { Cluster 1:19 } \\
\text { Cluser2:20 }\end{array}$ & 378.3 & 0.2777 & 0.4195 \\
\hline & $\begin{array}{l}\text { Cluster 1:13 } \\
\text { Cluster 2:13 } \\
\text { Cluster 3:13 }\end{array}$ & 587.1 & 0.1877 & 0.3608 \\
\hline & $\begin{array}{l}\text { Cluster 1:9 } \\
\text { Cluster 2:16 } \\
\text { Cluster 3:14 }\end{array}$ & 1081 & 0.0036 & 0.2198 \\
\hline
\end{tabular}

\subsection{Computational efficiency analysis}

As compared with global search partitioning methods, our proposed approach turns out to be more efficient by significantly decreasing the problem dimension. With the employment of network Laplacian spectrum, the search space of full network dataset is converted into pair-wise heuristic indicator (associated with a semi-optimal partition) including $\left(k_{\mathrm{MW}} k_{\mathrm{MVar}}\right)_{\max } / 2$ times of evaluations. In order to achieve Pareto optimality, the typical procedure for $k$-means clustering approach is repeatedly evaluating the partitioning solutions by using cluster validity indices (e.g. Davies-Bouldin index (DBI) based on Davies-Bouldin criterion [31]). The optimal clustering is determined through constantly testing predefined cardinalities from 2 to $\lfloor\sqrt{n}\rfloor$, where $n$ is the number of data samples. Distinguished from $k$-means algorithm, a well-trained SOM can efficiently produce network partitions with specific visualisation techniques.

Overall, the major computational task of the proposed approach includes construction of eigenspace, SOM based clustering and heuristic optimisation. For the first part, computing eigenvectors of $L_{\mathrm{MW}}$ and $L_{\mathrm{MVar}}$ normally takes $O\left(|N|^{3}\right)$ time. The time complexity can still be reduced by utilising sparse techniques for Laplacian matrix. Second, the total time complexity of training SOM is nearly $O((T+1) I)$, where $T$ is the number of neurons and $I$ is the number of iterations. In addition, automated clustering takes $O(T)$ time. Thus, the time complexity for the second part is $O((T+2) I)$. In conjunction with heuristic optimisation, the total time complexity is $\left.O\left((T+2) I k_{\mathrm{MW}} k_{\mathrm{MVar}}\right)_{\max } / 2\right)$. Such degree of time complexity makes the proposed partitioning method promising and competitive for practical applications.

\section{Experiments and performance}

In this section, the proposed approach is tested on three different systems i.e. New England 39-bus, IEEE 118-bus and Polish 2383-bus transmission systems. Comparative experiments are carried out with classical $k$-means algorithm. MATLAB SOM toolbox is used for SOM training.

\subsection{New England 39-bus system}

This test system consists of 39 buses, 10 generators and 46 branches. In this case, two operating conditions are considered including normal-loading and heavy-loading.

5.1.1 Parameter setting: In normal operating condition, the total generation is $6297.87 \mathrm{MW}$ and 1274.94 MVAr, and the total load is 6254.23 MW and 1387.1 MVAr. In heavy loading condition, the system is operated under a total generation of 7044.46 MW and 2020.11 MVAr, and a total load of 7004.23 MW and 2136.10 MVAr. Power flow solution is calculated based on the typical New England 39-bus system datasets [32].
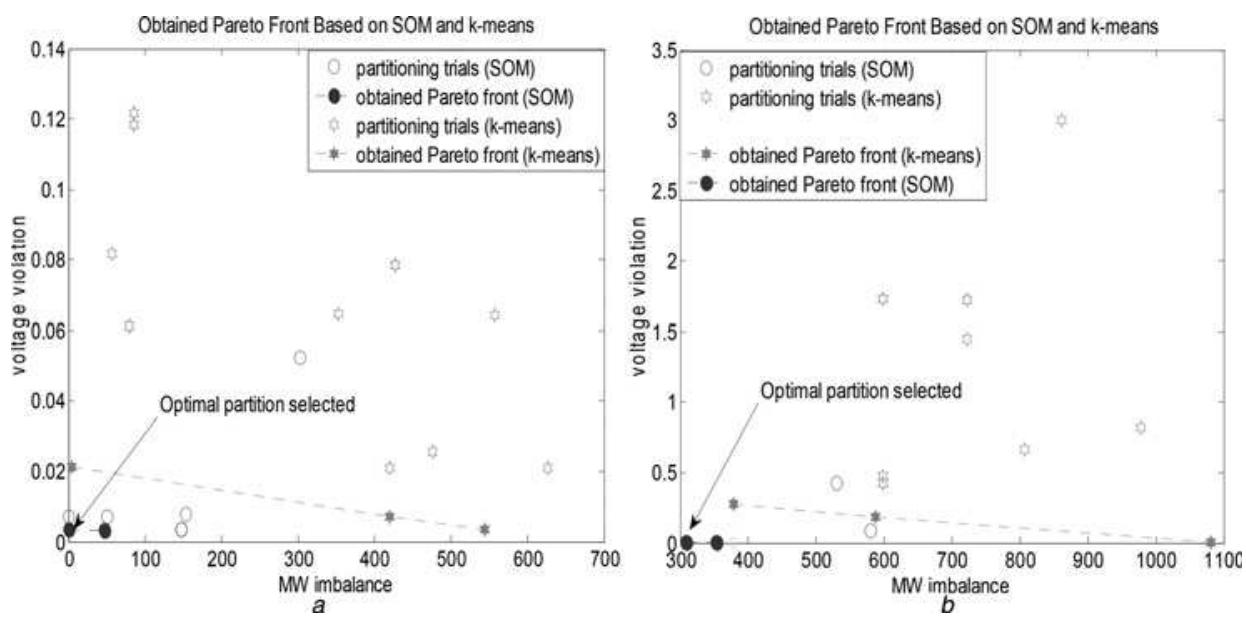

Fig. 7 Partitioning candidates

$a$ Heuristic partitioning results based on SOM and $k$-means in normal operating condition

$b$ Heuristic partitioning results based on SOM and $k$-means in heavy load operating condition 


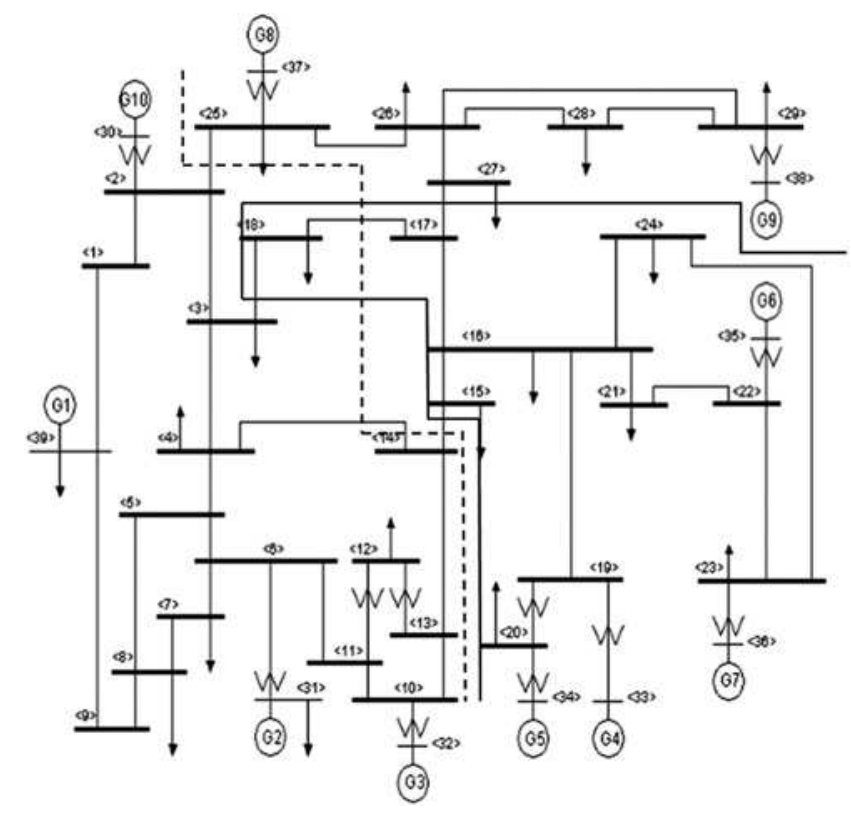

Fig. 8 New England 39-bus system partitioning boundaries on normal (solid line) and heavy load (dash line) operating conditions

In this case, one trains a $3 \times 3$ hexagonal Kohonen map by using MATLAB SOM toolbox (with a Gaussian neighbourhood function). As a comparative experiment, $k$-means algorithm (in MATLAB Statistics Toolbox) is used to cluster combined eigenspace. The clustering performance is evaluated by DBI. The optimal cardinality is selected with minimum DBI.

5.1.2 Network partitioning: According to the methodology presented in Section 4, different SOMs are first trained under two operating conditions. To demonstrate the automated clustering of SOM neurons, two given SOMs, which are trained, respectively, by eigenspace with $\left(k_{\mathrm{MW}}=1, k_{\mathrm{MVAr}}=2\right)$ and $\left(k_{\mathrm{MW}}=3, k_{\mathrm{MVAr}}=2\right)$ in normal operating condition, are clustered based on CONN linkage. Comparative experiment results are shown in Fig. 6 and Table 1.

Each point plotted in Figs. $6 a$ and $b$ represents the corresponding bus in this system, whose coordinates are determined by the first three eigenvectors in the combined eigenspace. Apparently, clustering results of SOM and $k$-means are fairly different. As for the $k$-means algorithm, the number of clusters is determined by DBI, which has a preference of four in this case. Based on the proposed approach, two clusters with the highest CONN_index are found.

As reported in Table 1, clusters obtained by the proposed approach have less real power imbalance and total voltage violation. Moreover, when more clustering information of real power are considered (i.e. $k_{\mathrm{MW}}=3, k_{\mathrm{MVAr}}=2$ ), the resultant clustering has a bias for less real power imbalance based on the proposed approach. In contrast, the $k$-means based clustering fails to capture such data features since larger MW imbalance is undesirably obtained under the condition of $k_{\mathrm{MW}}=3, k_{\mathrm{MVAr}}=2$. Obviously, SOM based clustering outperforms $k$-means because SOM can find an optimal distribution of the neuron vectors in the combined eigenspace (which is an adaptive vector quantisation process) such that the density distribution of the clustering information for real and reactive power can be best approximated. Therefore, SOM is more suitable for the proposed heuristic partitioning approach.

Based on the proposed heuristic algorithm, experimental comparisons of SOM based automated clustering and $k$-means are reported in Table 2. Partitioning candidates are shown in Fig. 7, where the obtained Pareto fronts are marked by dash lines.

As shown in Figs. $7 a$ and $b$, SOM based heuristic partitioning is more effective than $k$-means because less partitioning trials are exploited and the obtained Pareto front dominates the one obtained by $k$-means. This is reasonable since $k$-means based clustering fails to capture the data topology which combines information of real and reactive power (this has been proven through the whole experiment and an example is shown in Table 1). Among all partitioning alternatives (reported in Table 2), optimal partitions are selected with maximum fuzzy membership values, which are marked by asterisks. Partitioning boundaries are marked in Fig. 8.

\subsection{IEEE 118-bus system}

The IEEE 118-bus test system is extracted from the Midwestern US power grid in 1962, which consists of 54 generators and 186 branches. In this case, three operating conditions with different loading levels are considered.

5.2.1 Parameter setting: Three operating conditions with total load (4242 MW, 1438 MVAr), (5302.5 MW, 1840.6 MVAr), and (6151 MW, 2157 MVAr) are taken into account. Power flow solutions are produced based on typical IEEE 118-bus system datasets [24]

In this case, a $4 \times 4$ hexagonal Kohonen map is trained by using MATLAB SOM toolbox (with a Gaussian neighbourhood function), the $k$-means algorithm (in MATLAB Statistics Toolbox) is applied to compare the clustering results with the proposed approach.
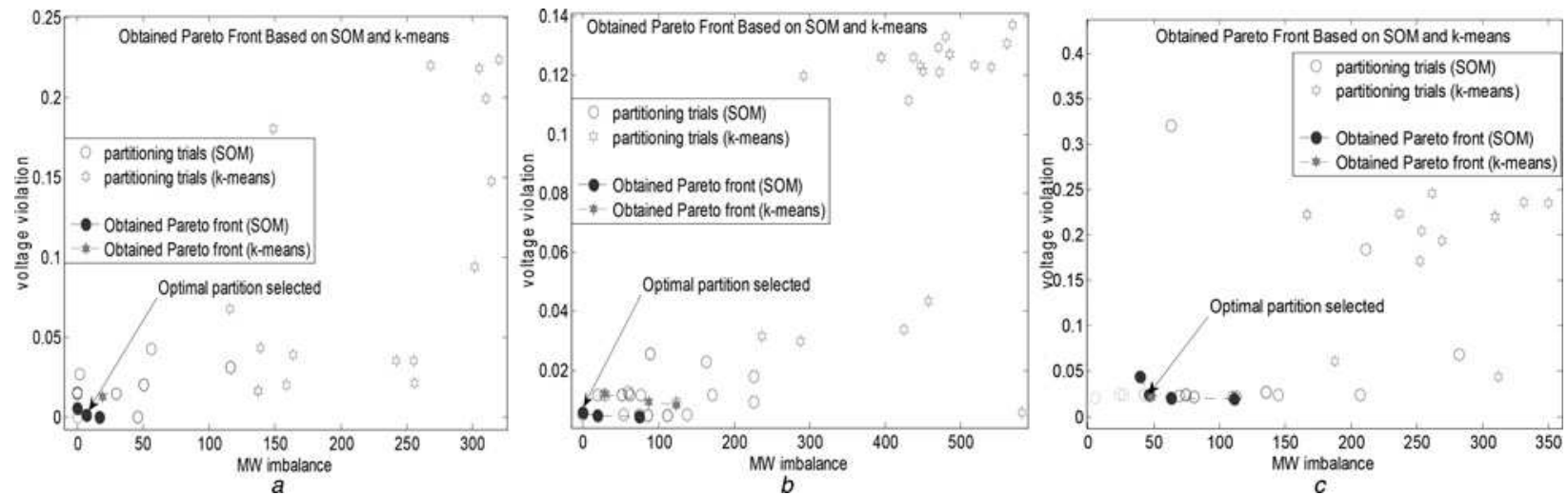

Fig. 9 Heuristic partitioning results based on SOM and k-means with total load

a $4242 \mathrm{MW}, 1438 \mathrm{MVAr}$

b $5302.5 \mathrm{MW}, 1840.6 \mathrm{MVAr}$

c $6151 \mathrm{MW}, 2157 \mathrm{MVA}$ 
Table 3 Partitioning results (IEEE 118-bus system)

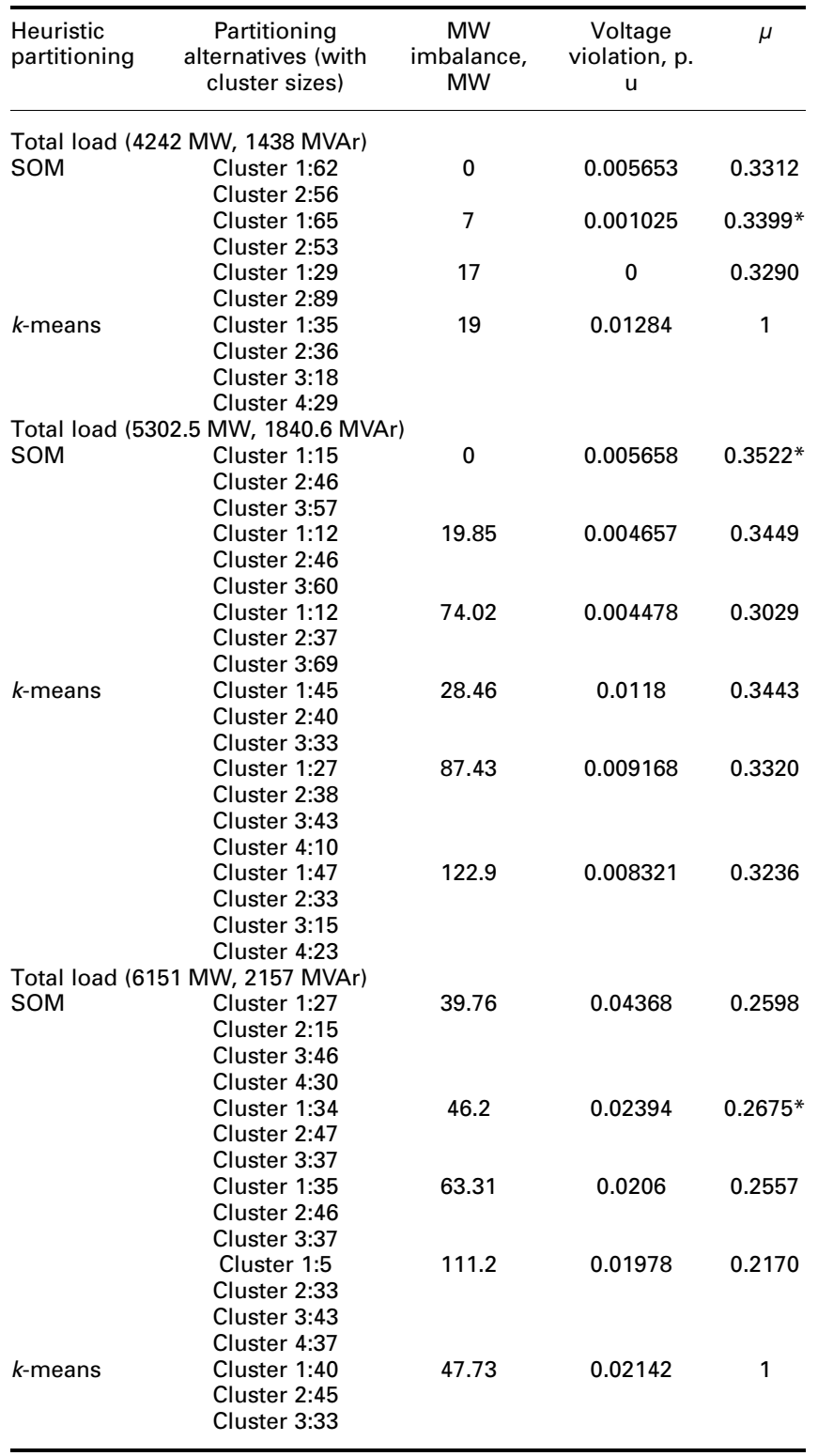

5.2.2 Network partitioning: In this case, along with the increasing of network size, the complexity of data topology (i.e. combined eigenspace) is significantly increased. Experimental results are shown in Fig. 9 and Table 3.

As shown in Fig. 9 and Table 3, the proposed approach outperforms $k$-means based clustering. Obviously, more partitioning trials are needed for $k$-means based clustering. With the increasing size of $\mathrm{PN}$, the proposed approach still performs well with less partitioning trials and better obtained Pareto front.

\subsection{Polish 2383-bus transmission system}

To provide further testing of the proposed approach, a realistic and relatively large transmission system, the 2383-bus transmission network data from Poland grid is used and two realistic loading profiles are considered in this case. This transmission network consists of 327 generators and 2896 branches.

5.3.1 Parameter setting: Two operating conditions with different load profiles are considered, i.e. (i) total generation of $25,280.97 \mathrm{MW}, 8810.45 \mathrm{MVAr}$, and total load of 24,558.38 MW, 8143.92 MVAr; and (ii) total generation of 18,961 MW, 6607.8 MVAr, and total load of 18,419 MW, 6108 MVAr.

A $6 \times 6$ hexagonal Kohonen map is trained by using MATLAB SOM toolbox (with a Gaussian neighbourhood function), $k$-means algorithm (in MATLAB Statistics Toolbox) is also applied on this transmission network to compare the clustering results.

5.3.2 Network partitioning: As shown in Fig. 10, with the complexity of data topology considerably increased, the proposed approach incorporating SOM based automated clustering is substantially more effective than $k$-means. As reported in Table 4 , it is obvious that $k$-means based clustering with employment of DBI is incapable of achieving satisfactory clustering boundaries that meet the objectives of minimising real power imbalance and voltage violation. As shown in Figs. $10 a$ and $b$, also reported in Table 4, the Pareto optimal solutions produced by the proposed approach have completely dominated the ones obtained by $k$-means.

5.4 Elapsed time: All case studies were repeated for 50 times on the workstation (Dell Precision T7600, Intel Xeon CPU E5-2667 0 (a) $2.90 \mathrm{GHz}, 2$ processors). The average computing time for each case is reported in Table 5. Such level of elapsed time is competitive, which shows high potential for practical applications.
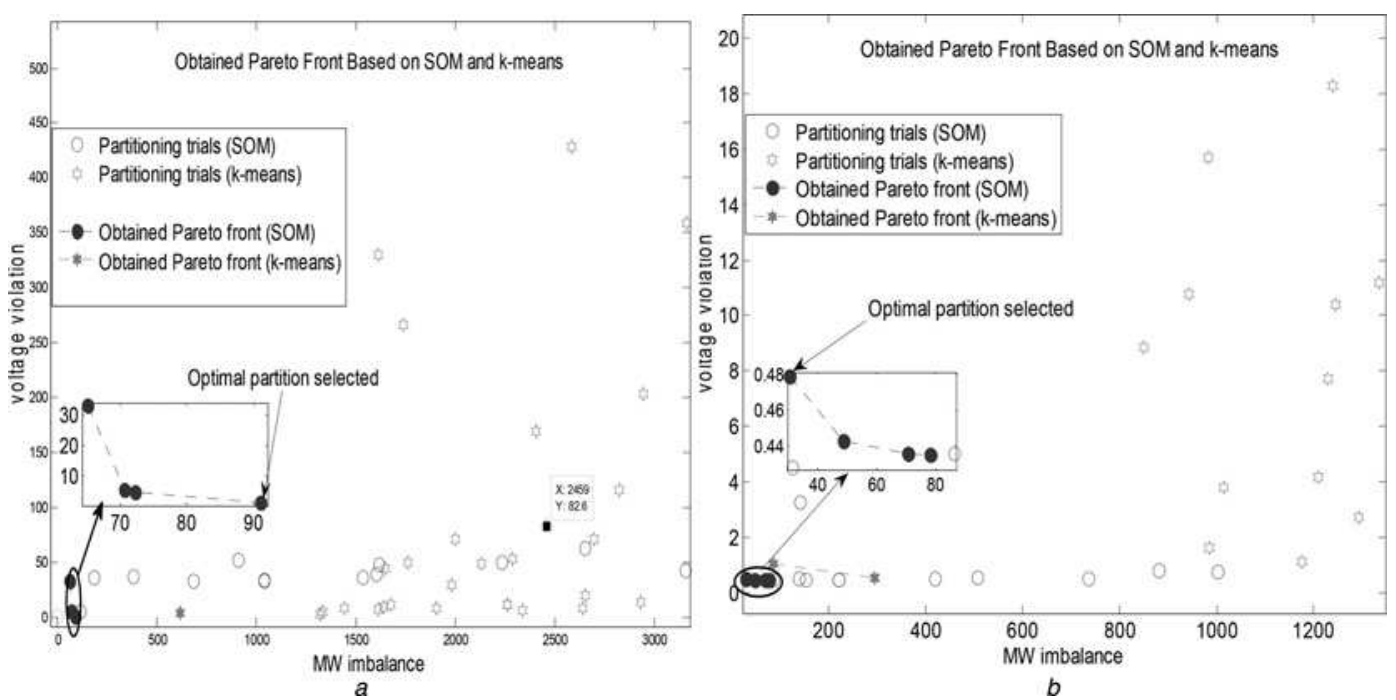

Fig. 10 Heuristic partitioning results based on SOM and k-means with total load a 24558.38 MW, 8143.92 MVAr

$b$ 18,419 MW, $6108 \mathrm{MVAr}$ 
Table 4 Partitioning results (Polish 2383-bus system)

\begin{tabular}{|c|c|c|c|c|}
\hline $\begin{array}{l}\text { Heuristic } \\
\text { partitioning }\end{array}$ & $\begin{array}{l}\text { Partitioning } \\
\text { alternatives (with } \\
\text { cluster sizes) }\end{array}$ & $\begin{array}{c}\text { MW } \\
\text { imbalance, } \\
\text { MW }\end{array}$ & $\begin{array}{c}\text { Voltage } \\
\text { violation, p. } \\
\text { u }\end{array}$ & $\mu$ \\
\hline \multicolumn{5}{|c|}{ Total load (24558.38 MW, 8143.92 MVAr) } \\
\hline \multirow[t]{4}{*}{ SOM } & $\begin{array}{c}\text { Cluster 1:24 } \\
\text { Cluster 2:1894 } \\
\text { Cluster } 3: 465\end{array}$ & 65.21 & 32.89 & 0.2146 \\
\hline & $\begin{array}{c}\text { Cluster } 1: 82 \\
\text { Cluster } 2: 464 \\
\text { Cluster } 3: 1153 \\
\text { Cluster } 4: 684\end{array}$ & 70.73 & 5.315 & 0.2595 \\
\hline & $\begin{array}{c}\text { Cluster 1:465 } \\
\text { Cluster 2:1153 } \\
\text { Cluster 3:765 }\end{array}$ & 72.31 & 4.56 & 0.2607 \\
\hline & $\begin{array}{c}\text { Cluster 1:466 } \\
\text { Cluster 2:1917 }\end{array}$ & 91.09 & 1.237 & $0.2653 *$ \\
\hline $\begin{array}{l}\text { k-means } \\
\text { Total load (1 }\end{array}$ & $\begin{array}{c}\text { Cluster 1:521 } \\
\text { Cluster 2:70 } \\
\text { Cluster 3:723 } \\
\text { Cluster 4:1069 } \\
419 \text { MW, 6108 MVAr }\end{array}$ & 619 & 3.795 & 1 \\
\hline \multirow[t]{4}{*}{ SOM } & $\begin{array}{c}\text { Cluster 1:58 } \\
\text { Cluster 2:2325 }\end{array}$ & 30.62 & 0.479 & $0.2521^{*}$ \\
\hline & $\begin{array}{l}\text { Cluster 1:347 } \\
\text { Cluster 2:2036 }\end{array}$ & 48.72 & 0.4427 & 0.2513 \\
\hline & $\begin{array}{c}\text { Cluster 1:350 } \\
\text { Cluster 2:2033 }\end{array}$ & 70.94 & 0.4352 & 0.2488 \\
\hline & $\begin{array}{c}\text { Cluster 1:429 } \\
\text { Cluster 2:1944 }\end{array}$ & 78.37 & 0.4348 & 0.2478 \\
\hline \multirow[t]{2}{*}{$k$-means } & $\begin{array}{c}\text { Cluster 1:852 } \\
\text { Cluster 2:1277 } \\
\text { Cluster 3:254 }\end{array}$ & 86.11 & 1.032 & 0.5183 \\
\hline & $\begin{array}{c}\text { Cluster 1:395 } \\
\text { Cluster 2:960 } \\
\text { Cluster 3:1028 }\end{array}$ & 295.9 & 0.5156 & 0.4817 \\
\hline
\end{tabular}

Table 5 Elapsed time in different cases

\begin{tabular}{lcc}
\hline Test case & Load profiles & $\begin{array}{c}\text { Averaging elapsed } \\
\text { time, s }\end{array}$ \\
\hline IEEE 39-bus system & 6254.23 MW 1387.1 & 6.241 \\
& MVAr & 6.140 \\
& 7044.46 MW 2020.11 & \\
IEEE 118-bus system & MVAr & 8.126 \\
& 5302.5 MW, 1438 MVAr & 8.233 \\
Polish 2383-bus & 6151 MW, 2157 MVAr & 8.569 \\
system & 24,558.38 MW, 8143.92 & 89.324 \\
& MVAr & 93.605 \\
\hline
\end{tabular}

\section{Conclusions}

An adaptive hybrid partitioning approach is proposed in this paper, which combines Laplacian spectrum of $\mathrm{PNs}$ and SOM based automated clustering. A tailored heuristic optimisation algorithm is also developed to solve the bi-objective partitioning problem. Case studies are carried out based on the New England 39-bus, IEEE 118-bus and Polish 2383-bus transmission systems, which demonstrate the effectiveness and computational efficiency of the proposed approach, in producing optimal self-sufficient network partitions with small real power imbalance and satisfactory voltage profiles.

This hybrid partitioning approach can layout a useful groundwork for the development of self-healing smart grid. With the advantages of SOM, the approach can be easily extended by taking other operating factors (e.g. generator coherency and voltage angle) in to considerations. The promising partitioning results obtained in this paper have demonstrate the effectiveness of SOM and other relevant learning algorithms for applications in smart grid planning and operation control.

\section{Acknowledgments}

This work was partially supported by Research Grants Council of Hong Kong, China under grant no. T23-407/13N and T23-701/14N.

\section{References}

1 Arefifar, S.A., Mohamed, Y.A.R.I., Fouly, T.H.M.E.: 'Comprehensive operational planning framework for self-healing control actions in smart distribution grids', IEEE Trans. Power Syst., 2013, 28, (4), pp. 4192-4200

2 Zidan, A., El-Saadany, E.F.: 'A Cooperative multiagent framework for self-healing mechanisms in distribution systems', IEEE Trans. Smart Grid, 2012, 3, (3), pp. $1525-1539$

3 Vaccaro, A., Loia, V., Formato, G., et al.: 'A self-organizing architecture for decentralized smart microgrids synchronization, control, and monitoring', IEEE Trans. Ind. Inf., 2015, 11, (1), pp. 289-298

4 Loia, V., Furno, D., Vaccaro, A.: 'Decentralised smart grids monitoring by swarm-based semantic sensor data analysis', Int. J. Syst. Control Commun., 2013, 5, (1), pp. 1-14

5 Saleh, A.O.M., Laughton, M.A.: 'Cluster analysis of power-system networks for array processing solutions', IEE Proc. C Gener. Transm. Distrib., 1985, 132, pp. 172-178

6 Yurong, W., Fangxing, L., Qiulan, W., et al.: 'Reactive power planning based on fuzzy clustering, gray code, and simulated annealing', IEEE Trans. Power Syst., 2011, 26, (4), pp. 2246-2255

7 Bjorndal, M., Jornsten, K.: 'Zonal pricing in a deregulated electricity market', Energy J., 2001, 22, pp. 51-73

8 Donde, V., Lopez, V., Lesieutre, B., et al.: 'Identification of severe multiple contingencies in electric power networks'. Proc. 37th Annual North American Power Symp., 2005, 2005

9 Burger, M., Zelazo, D., Allgower, F.: 'Hierarchical clustering of dynamical networks using a saddle-point analysis', IEEE Trans. Autom. Control, 2013, 58, (1), pp. 113-124

10 Yang, B., Vittal, V., Heydt, G.T.: 'Slow-coherency-based controlled Islanding - a demonstration of the approach on the August 14, 2003 blackout scenario', IEEE Trans. Power Syst., 2006, 21, (4), pp. 1840-1847

11 Xie, N., Torelli, F., Bompard, E., et al.: 'A graph theory based methodology for optimal Pmus placement and multiarea power system state estimation', Electr. Power Syst. Res., 2015, 119, pp. 25-33

12 Sun, L., Zhang, C., Lin, Z., et al.: 'Network partitioning strategy for parallel power system restoration', IET Gener. Transm. Distrib., 2016, 10, (8), pp. 1883-1892

13 Ding, T., Sun, K., Huang, C., et al.: 'Mixed-integer linear programming-based splitting strategies for power system islanding operation considering network connectivity', IEEE Syst. J., 2015, PP, (99), pp. 1-10

14 Cotilla-Sanchez, E., Hines, P.D.H., Barrows, C., et al.: 'Multi-attribute partitioning of power networks based on electrical distance', IEEE Trans. Power Syst., 2013, 28, (4), pp. 4979-4987

15 Ding, L., Gonzalez-Longatt, F.M., Wall, P., et al.: 'Two-step spectral clustering controlled Islanding algorithm', IEEE Trans. Power Syst., 2013, 28, (1), pp. 75-84

$16 \mathrm{Li}$, J., Liu, C.C., Schneider, K.P.: 'Controlled partitioning of a power network considering real and reactive power balance', IEEE Trans. Smart Grid, 2010, 1, (3), pp. 261-269

17 Chopade, P., Bikdash, M.: 'New centrality measures for assessing smart grid vulnerabilities and predicting brownouts and blackouts', Int. J. Crit. Infrastruct. Prot., 2016, 12, pp. 29-45

18 Pinar, A., Meza, J., Donde, V., et al.: 'Optimization strategies for the vulnerability analysis of the electric power grid', SIAM J. Optim., 2010, 20, (4), pp. 1786-1810

19 Luxburg, U.: 'A tutorial on spectral clustering', Stat. Comput., 2007, 17, (4), pp. $395-416$

20 Davis, C., Kahan, W.M.: 'The rotation of eigenvectors by a perturbation. Iii', SIAM J. Numer. Anal., 1970, 7, (1), pp. 1-46

21 Shi, J., Malik, J.: 'Normalized cuts and image segmentation', IEEE Trans. Pattern Anal. Mach. Intell., 2000, 22, (8), pp. 888-905

22 Schloegel, K., Karypis, G., Kumar, V.: 'A new algorithm for multi-objective graph partitioning*' (Springer, 1999)

23 Kohonen, T.: 'The self-organizing map', Proc. IEEE, 1990, 78, (9), pp. 1464-1480

24 Tasdemir, K., Milenov, P., Tapsall, B.: 'Topology-based hierarchical clustering of self-organizing maps', IEEE Trans. Neural Netw., 2011, 22, (3), pp. 474-485

25 Vesanto, J., Alhoniemi, E.: 'Clustering of the self-organizing map', IEEE Trans. Neural Netw., 2000, 11, (3), pp. 586-600

26 Murtagh, F.: 'Interpreting the Kohonen self-organizing feature map using contiguity-constrained clustering', Pattern Recogn. Lett., 1995, 16, (4), pp. 399-408

27 Tasdemir, K., Merényi, E.: 'Data topology visualization for the self-organizing map'. ESANN, 2006

28 Tasdemir, K., Merenyi, E.: 'Exploiting data topology in visualization and clustering of self-organizing maps', IEEE Trans. Neural Netw., 2009, 20, (4), pp. 549-562

29 Deb, K., Pratap, A., Agarwal, S., et al.: 'A fast and elitist multiobjective genetic algorithm: Nsga-Ii', IEEE Trans. Evol. Comput., 2002, 6, (2), pp. 182-197

30 Agrawal, S., Panigrahi, K.B., Tiwari, M.K.: 'Multiobjective particle swarm algorithm with fuzzy clustering for electrical power dispatch', IEEE Trans. Evol. Comput., 2008, 12, (5), pp. 529-541

31 Davies, D.L., Bouldin, D.W.: 'A cluster separation measure', IEEE Trans. Pattern Anal. Mach. Intell., 1979, PAMI-1, (2), pp. 224-227

32 'Power Flow Cases'. Available at http://icseg.iti.illinois.edu/power-cases 